\title{
Diabetes Management: Expectations and Limitations
}

\author{
Neeta Raj Sharma ${ }^{1}$ and Gundu H R Rao ${ }^{2 *}$
}

${ }^{1}$ School of Biotechnology and Biosciences, Lovely Professional University, Phagwara, India

${ }^{2}$ Laboratory Medicine and Pathology, University of Minnesota, Minneapolis, Minnesota, USA

Cardiometabolic diseases, including obesity and Type- 2 diabetes, are a growing concern and have become an epidemic worldwide [14]. World Health Organization (WHO) Global prevalence of Type-2 diabetes estimates, incidence of this disease, to be $9 \%$ among adults. An estimated 1.5 million death worldwide, were directly caused by diabetes. More than $80 \%$ of the type- 2 diabetes related deaths occur in low-and middle-income countries. Worldwide in 2013, it was estimated that almost 382 million people suffer from diabetes and this is supposed to reach a high of 600 million by 2035. According to the World Health Organization estimates, the impact of Type-2 diabetes related health care expenditure, to prevent and manage diabetes and its clinical complications in USA alone, was USD 360 billion in 2010 and will exceed 360 billion by 2030 [5]. WHO predicts losses in national income from Diabetes and Cardiovascular Disease (CVD) to be in equivalents of 557 billion in International Dollars (ID) in China, 303 billion in Russia and 236 billion in India [5]. Currently there are 65 million diabetics in India and an equal number of pre-diabetics. Incidence of type- 2 diabetes in China also has reached epidemic proportions. Gulf Countries also have reported a high incidence of diabetes. One of the Global targets for Diabetes management was to halt by 2025 , the rise in the age standardized adult prevalence of this disease at its 2010 levels [1]. However, according a recent publication in the Lancet (April 2016) by the NCD Risk Factor Collaboration, if the post-2000 trends continue in the incidence and rise of type- 2 diabetes, the probability of meeting the global target of halting the rise in the prevalence of diabetes by 2025 at the 2020 level worldwide is lower than $1 \%$ for men and women. In this mini-review, we discuss some aspects of the expectations and limitations, as it relates to Diabetes Management. For additional information on this complex subject, readers are urged to refer to comprehensive reviews on the subject.

Overweight and obesity are the major driving forces for Global diabetic epidemic. Current worldwide estimate of number of overweight's (BMI>25 $\mathrm{Kg} / \mathrm{m}^{2}$ ) will increase from 1.3 billion to 2.0 billion by 2030 . Having said that, a tendency toward greater abdominal obesity and loss of muscle mass among Asians result in increased insulin resistance compared to the Western population. This "metabolically obese (visceral obesity)" phenotype among normalweight individuals in Asia, may explain the increased predisposition for diabetes, despite low prevalence of "obesity" in Asia. A collaborative study between researchers at the University of Minnesota and the staff of Madras Diabetes Research Foundation, Chennai, India, demonstrated this differential distribution of fat in Asian subjects compared to Caucasians, Afro-Americans and Hispanic populations [6]. Major metabolic contributors for the development of this chronic disorder are hyperglycemia, insulin resistance, dyslipidemia, hypertension, endothelial dysfunction, obesity, oxidative stress, and metabolic syndrome. Studies done at several research centers indicates, that this chronic disease by and large is preventable by diet, exercise and life style modification [6-8]. Professor Roy Taylor of Newcastle University has demonstrated that even well characterized cases of type2 diabetes can be reversed by very low calorie diets [9]. A recent study published by the same group showed that type- 2 diabetes could be kept in remission 6 months after the low-calorie diet was completed.
However, the researchers are not sure of the long-term effects of such a low calorie diet or as to how long they have to be kept o such a low calorie diet to maintain the remission of the type- 2 diabetes (www. dibetes.uk:low-clorie).

By and large, clinicians and health care workers, who manage diabetes patients, firmly believe, that the early detection of the risks for CMDs and effective management of observed risks is the best choice, for reducing the Global burden of these metabolic diseases. What is the earliest stage that we can start prevention strategies? According to the seminal epidemiological work at the Mission Hospital, Mysore, India, thirty percent of the children born in India, are of low birth weight. The birth records of all the children born in this hospital are maintained from 1936. In view of this meticulous record keeping, Medical Research Council (MRC) of UK has set up an epidemiology wing at this hospital. Studies from this group on "Mysore Cohort" for decades, have established that these children as they grow up have a higher risk for developing metabolic diseases, such as hypertension, obesity, metabolic syndrome, heart disease and stroke [2,4]. Close to thirty million children are born in India every year. That means, we add 10 million children a year to our population every year, who are "at risk", for developing cardiometabolic disorders. These findings are not unique to South Indian children. Studies on the effect of low birth weight on the development of metabolic syndrome, has been investigated in the Fetal Origin of Adult Disease (FOAD) "cohort" of Beijing, China [2]. Several studies worldwide, have demonstrated that this condition is preventable by appropriate intervention and preventive strategies. More than half of the world's low birth weight children are born in South Asia. According to experts, preventive strategies that combine nutrition-based interventions that improve women's health status are needed to reduce this healthcare burden [8].

Diabetes is a chronic metabolic disease, in which altered glucose metabolism plays a critical role. Associated abnormalities in protein, carbohydrate and fat metabolism are the result of altered insulin action on peripheral gut tissue due to insufficient insulin secretion. Obesity is an important modulator of various risks associated with vascular diseases, including insulin resistance. The association of excess weight with type-2 diabetes and insulin resistance is well recognized [47]. Insulin resistance is also linked with a variety of associated risks including hypertension, hyper-lipidemia, atherosclerosis, metabolic syndrome, and endothelial dysfunction. In a study in UK it was demonstrated, that each 2 hour increment in television watching was

*Corresponding author: Gundu H. R. Rao, Emeritus Professor, Laboratory Medicine and Pathology, University of Minnesota, Minneapolis, Minnesota, USA Tel: 9525945248; E-mail: gundurao9@gmail.com

Received March 11, 2016; Accepted April 20, 2016; Published April 27, 2016

Citation: Sharma NR, Rao GHR (2016) Diabetes Management: Expectations and Limitations. J Diabetes Metab 7: 662. doi:10.4172/2155-6156.1000662

Copyright: @ 2016 Sharma NR, et al. This is an open-access article distributed under the terms of the Creative Commons Attribution License, which permits unrestricted use, distribution, and reproduction in any medium, provided the original author and source are credited. 
associated with $14 \%$ increase in diabetes risk. Studies at the Diabetes Center at Leicester University (personal communication), England, have demonstrated that 6 hours of standing (during working hours) burns 300 calories (equivalent to 2 hrs walking on treadmill). All Gulf countries have very high incidence of type-2 diabetes. According to the international Federation Guidelines, there is increasing evidence that supports the importance of controlling or managing all the three components of the glucose triad; hemoglobin A1c, Fasting Glucose, and Post Prandial Glucose [10]. Post-meal glycemia (PMG) is defined as $140 \mathrm{mg}$ of glucose/dl, $2 \mathrm{hr}$ after the ingestion of a meal. The PMG is a major contributor for complications associated with diabetes, as such targeting PMG, as a management strategy will help reduce the overall risk for developing clinical complications of diabetes such as peripheral neuropathy, nephropathy, retinopathy and cardiovascular problems.

Americans on an average consume 250 to 300 gms of carbs a day, accounting to $55 \%$ of their daily intake. South Asians by and large, take lot more of carbs as their caloric intake. All carbohydrates get converted into sugar in the blood, the more refined the carbs are, the quicker the conversion occurs. In a healthy adult the metabolism works fine, however, overtime the metabolism changes and insulin resistance develops. Studies done in several laboratories, have demonstrated the beneficial effect of Mediterranean and low carb diet, compared to the low fat diet. In a 12 week study, Prof. Stephen Phinney (Emeritus Professor of Nutrition, at UC Davis, USA) and his associates put 40 subjects with metabolic syndrome on a 1500-calorie diet. Half of the subjects were on low fat and high carb diet, other half on low-carb and high fat diet. Despite the high fat that the low carb diet group was getting, at the end of the study levels of triglycerides had dropped by $50 \%$ in this group. Levels of good cholesterol (HDL) increased by $15 \%$. The researchers concluded that contrary to what one may expect, dietary fat intake was not directly related to blood fat. Rather the amount of carbohydrates in the diet appears to be a potent contributor. "Fat is not the problem," says Dr. Walter Willett, Chairman of the Department of Nutrition at the Harvard School of Public Health (LA Times, 2/20/2016). "If Americans could eliminate sugary beverages, potatoes, white bread, pasta, white rice and sugary snacks, we would wipe out almost all the problems we have with excess weight and diabetes and other metabolic diseases." A meta-analysis that included randomized controlled studies found that low carb, non-energy restricted diets appear to be at least as effective as low-fat energy-restricted diets, in inducing weight loss for up to one year. When discussing about the diet and diabetes, one can conclude that lowering the glycemic index and the absolute amount of carbohydrates in the diet, can have a profound effect upon lowering the blood glucose; whereas dietary protein has very little effect on blood glucose and dietary fat has none [9-15].

In developing countries less than half of people with diabetes are diagnosed [1-4]. Type-2 diabetes can remain undetected for many years and the diagnosis by and large made from associated complications, such as elevated blood or urinary sugar. Undiagnosed diabetes accounts for $85 \%$ of those diagnosed with this disease in South Africa, over $60 \%$ in India, $80 \%$ in Cameron, $70 \%$ in Ghana and over $80 \%$ in Tanzania. Type-2 diabetes is responsible for $85-95 \%$ of all diabetes, in high-income countries and may be even higher in developing countries [1]. Eighty percent of Type-2 diabetes is preventable by changing diet, increasing physical activity and improving the lifestyle. As a part of its Global mission to promote diabetes care and prevention, International Diabetes Federation develops guidelines, which review evidence to date on post-meal-glucose values and the development of clinical complications of diabetes [10]. These guidelines and recommendations are intended to help clinicians and professional organizations in developing strategies for effective management of PMG in diabetics. http://www.idf.org.

In the conference on Diabetes in Dubai $\left(6^{\text {th }}\right.$ Global Summit on diabetes Nov $4^{\text {th }}$ 2015), we presented our views on some of the novel approaches in early detection of risks associated with type 2-diabetes and its clinical complications [16,17]. The scientists and engineers of LD Technologies, Miami, Florida, have developed a non-invasive diagnostic platform for the diagnosis of complications associated with the progression of diabetes. This diagnostic platform called TMOxi in USA and as R.I.S.C.-Test in India (Lifespan, India), uses pulse oximeter, blood pressure monitor and galvanic skin response monitor, to obtain data from the subjects. Appropriate algorithms, convert the data obtained during these studies, to risk scores for various complications such as lipid biomarkers, IGT markers, blood pressure markers, endothelial function markers etc $[18,19]$. Indeed, the Lifespan India in their brochures and advertisement, claim that the R.I.S.C-test identifies over 30 cardiometabolic risks.

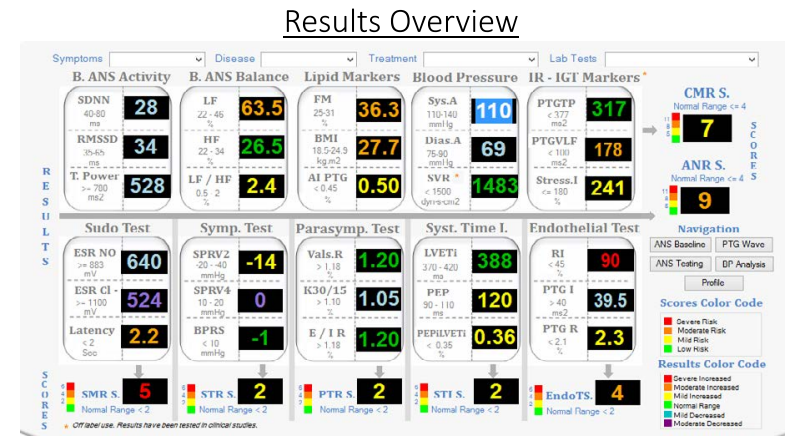

Courtesy: Dr. Albert Maarek, LD Technologies, Miami, Florida (www.ldteck.com).

However, if one takes a look at the results overview generated by this test, it becomes clear, that they are grouped under just two headings, cardiometabolic risk (CMR) score and Autonomic Nervous Response (ANR) score. The developers of this novel technology had great expectations in terms of its ability to detect a variety of risk scores. However, from the perspective of a clinician the scores and the values generated using this system do not provide a means to manage the therapy of this complicated disease. Having said that, we need to take into consideration the novelty of cutting edge technologies like this, which provide us risk prediction capabilities. This platform clearly demonstrates the superiority of software, analytics and algorithmsbased clinical decision support. Appropriate use of this and other technologies, collection and collation of massive clinical data, analysis of such data, will provide us new and useful information for detection of early lesions that develop as the disease progresses.

Type-2 diabetes is a major growing concern worldwide. By and large, depending on the resources (financial as well as human), different countries have generally focused management of diabetes and its clinical complications, to normalizing overall glycemic control, as assessed by fasting glucose or hemoglobin Alc. This approach of management of type- 2 diabetes, only addresses the need of individuals who already are diabetic. This approach will not reduce the healthcare burden or the incidence of this chronic disease. Framingham risk studies for cardiovascular disease and various preventive measures including the changes in life style reduced cardiovascular morbidity and mortality in the developed countries of the world. Therefore, 
Citation: Sharma NR, Rao GHR (2016) Diabetes Management: Expectations and Limitations. J Diabetes Metab 7: 662. doi:10.4172/21556156.1000662

Page 3 of 3

there was great expectation, that diabetes and its clinical complications too, can be brought under control. Contrary to these expectations, the incidence of type- 2 diabetes in many countries has increased in epidemic proportions. In view of these observations, the best choice we have is, to go by the World Diabetes Federation recommendations and manage the glycemia efficiently. In addition, develop programs for early detection of altered glucose metabolism and implement robust strategies for normalization of this altered state. In conclusion, through robust prevention programs, reduce the disease incidence, through better diagnostic tests, detect early risks that initiate or promote clinical complications, and by effective management of the risks, reduce or prevent acute events related to end-organ failure.

\section{References}

1. Rao MV (2007) GHR: Diabetes Mellitus (Type-2): Epidemiology, Risk Management and Prevention. Jaypee Medical Publishers, New Delhi, India.

2. Rao GHR, Gandhi PG, Sharma V (2014) Clinical Complications of Type-2 Diabetes in South Asians and Chinese Populations: An Overview. J Diabetes Metab 5:420.

3. Rao GHR, Nagendra HR (2012) Holistic approach for the management of heart disease and type-2 diabetes. J Preve Cardiol 2:231-238.

4. Rao GHR (2015) Non-Traditional Approaches for Diagnosis and Management of Type-2 Diabetes Mellitus: Point of View. J Diabetes Metab 6: 489

5. WHO Statistical Information System (WHOSIS) (2008) World Health Statistics.

6. Bajaj HS, Pereira MA, Anjana M, Deepa R, Mohan V, et al. (2014) Gross M: Comparison of Relative Waist circumference between Asian Indian and US Adults. J Obesity

7. Rao GH, Thethi I, Fareed J (2011) Vascular disease: obesity and excess weight as modulators of risk. Expert Rev Cardiovasc Ther 9: 525-534.
8. Ramakrishnan U (2004) Nutrition and low birth weight: from research to practice. Am J Clin Nutr 79: 17-21.

9. Steven S, Lim EL, Taylor R (2013) Population response to information on reversibility of Type 2 diabetes. Diabet Med 30: e135-138.

10. Rao GHR (2011) Management of Type-2 diabetes by anti-platelet therapy with special reference to aspirin. Front Bio Sci 3: 1-15.

11. Ceriello A, Colagiuri S (2008) International Diabetes Federation guideline for management of postmeal glucose: a review of recommendations. Diabet Med 25: $1151-1156$.

12. Jenkins DJ, Wolever TM, Taylor RH, Barker H, Fielden H, et al. (1981) Glycemic index of foods: a physiological basis for carbohydrate exchange. Am J Clin Nutr 34: 362-366.

13. Ludwig DS (2002) The glycemic index: physiological mechanisms relating to obesity, diabetes, and cardiovascular disease. JAMA 287: 2414-2423.

14. Neill DF, Westman EC, Bernstein RK (2003) The effects of a low-carbohydrate regimen on glycemic control and serum lipids in diabetes mellitus. Metab Syndr Relat Disord 1: 233-237.

15. Westman EC, Yancy WS Jr, Mavropoulos JC, Marquart M, McDuffie JR (2008) The effect of a low-carbohydrate, ketogenic diet versus a low-glycemic index diet on glycemic control in type 2 diabetes mellitus. Nutr Metab (Lond) 5: 36.

16. Volek JS, Phinney SD, Forsythe CE, Quann EE, Wood RJ, et al. (2009) Carbohydrate restriction has a more favorable impact on the metabolic syndrome than a low fat diet. Lipids 44: 297-309.

17. Santos FL, Esteves SS, da Costa Pereira A, Yancy WS Jr, Nunes JP (2012) Systematic review and meta-analysis of clinical trials of the effects of low carbohydrate diets on cardiovascular risk factors. Obes Rev 13: 1048-1066.

18. Gandhi PG, Rao GH (2014) The spectral analysis of photoplethysmography to evaluate an independent cardiovascular risk factor. Int J Gen Med 7: 539-547.

19. Gandhi PG, Rao GHR (2015) Detection of Neuropathy using sudomotor tests in Type-2 diabetes. Degener Neurol Neuromuscul Dis 5:1-7. 